\title{
Menschenrechte: Kommunikation und Lebendes Recht
}

\author{
Dissertation \\ der Rechtswissenschaftlichen Fakultät \\ der Universität Zürich
}

zur Erlangung der Würde eines Doktors der Rechtswissenschaft

\author{
vorgelegt von \\ Hans Ulrich Locher \\ von Heiden AR \\ genehmigt auf Antrag von \\ Prof. Dr. iur. Manfred Rehbinder
}




\section{Inhaltsverzeichnis}

1. Einleitung $\ldots \ldots \ldots \ldots \ldots \ldots \ldots \ldots \ldots \ldots \ldots \ldots \ldots \ldots \ldots \ldots \ldots \ldots \ldots \ldots \ldots \ldots, 13$

1.1 Zur Entwicklung dieser Arbeit.......................... 13

1.2 Übersicht und Methodik............................... 15

1.3 Zum Titel: Begriffe und Verständnis .................... 16

2. Die Rezeption der Menschenrechte der Vereinten Nationen

in ausgewählten Zeitungen............................. 19

2.1 Das Übereinkommen vom 9. Dezember 1948 über die Verhütung und Bestrafung von Völkermord .......................... 20

2.2 Die Deklaration der Menschenrechte durch die Vereinigten Nationen

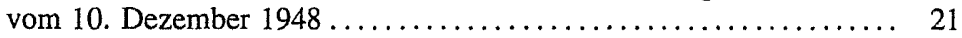

2.2.1 Kommentare................................... 21

2.2.2 Berichterstattung $\ldots \ldots \ldots \ldots \ldots \ldots \ldots \ldots \ldots \ldots \ldots \ldots, 22$

2.3. Das Übereinkommen über die Rechtsstellung der Staatenlosen vom

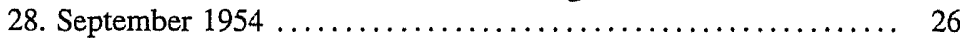

2.4 Das Übereinkommen zur Beseitigung jeder Form von Rassendiskrimi-

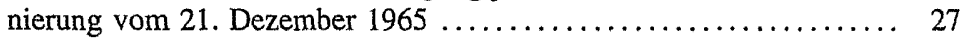

2.5 Die beiden Pakte der Vereinten Nationen vom 16. Dezember 1966... 28

2.6 Die weiteren Übereinkommen der Uno von menschenrechtlicher Relevanz ...................................... 31

2.6.1 Das Übereinkommen zur Beseitigung jeder Form von Diskriminierung der Frau vom 18. Dezember 1979............... 31

2.6.2 Das Übereinkommen gegen Folter und andere grausame, unmenschliche oder erniedrigende Behandlung oder Strafe vom 10. Dezember 1984

2.6.3 Übereinkommen über die Rechte des Kindes vom 20. November 1989

2.6.4 Übereinkommen zum Schutz der Rechte aller Wanderarbeitnehmer und ihrer Familienangehörigen vom 18. Dezember 1990 .. 32

2.7 Zusammenfassung $\ldots \ldots \ldots \ldots \ldots \ldots \ldots \ldots \ldots \ldots \ldots \ldots \ldots \ldots \ldots \ldots \ldots \ldots \ldots \ldots, 32$

3. Die Verbreitung der Menschenrechte nach nationalen Kriterien....... 35

3.1 Vorgeschichte bis zur Deklaration der Vereinten Nationen.......... 36

3.2 Die Nationalität der Kommissionsmitglieder ................. 38

3.3 Abstimmung über die Menschenrechtserklärung nach Nationen...... 40

3.4 Uno Pakt I und seine weltweite Verbreitung $\ldots \ldots \ldots \ldots \ldots \ldots \ldots, 42$

3.5 Uno Pakt II und seine weltweite Verbreitung.................. 45

3.6 Zusammenfassung $\ldots \ldots \ldots \ldots \ldots \ldots \ldots \ldots \ldots \ldots \ldots \ldots \ldots \ldots \ldots \ldots \ldots \ldots \ldots, 48$ 
4. Folgen der Menschenrechte und die Problematik des Systems ....... 52

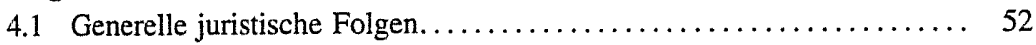

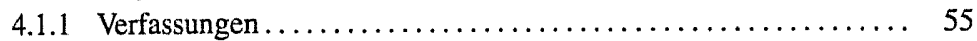

4.1.2 Verfahren, Rechtsmittel, Zwangsmassnahmen .......... 56

4.1 .3 Gerichte .............................. 57

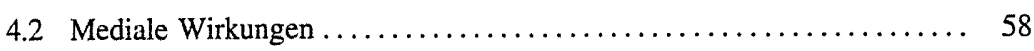

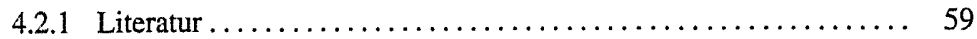

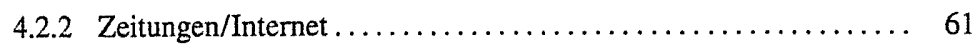

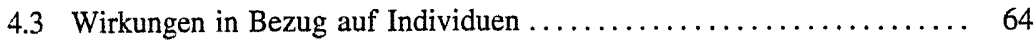

4.3.1 Praktisch umfassende Akzeptanz................... 64

4.3.2 Eher zwiespältige Resultate.................... 65

4.3.3 Differenz als Forschungsaufgabe $\ldots \ldots \ldots \ldots \ldots \ldots \ldots \ldots \ldots 66$

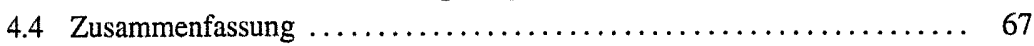

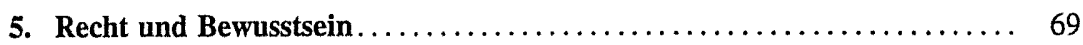

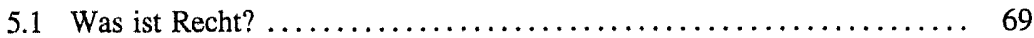

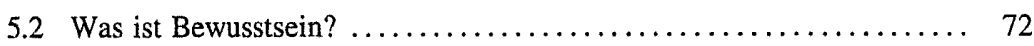

5.3 Vom Rechtsbewusstsein und den Rechtstatsachen .............. 75

5.3 .1 Individuelles Rechtsbewusstsein ................. 75

5.3 .2 Kollektives Rechtsbewusstsein ................. 76

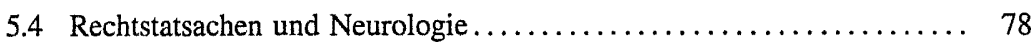

5.4.1 Rechtsneurologie statt Rechtsbewusstsein ............ 78

5.4 .2 Schuldfähigkeit und neurologische Erkenntnisse......... 79

5.4 .3 Statistik von Urteilen und Zurechnungsfähigkeit.......... 80

5.5 Zusammenfassung $\ldots \ldots \ldots \ldots \ldots \ldots \ldots \ldots \ldots \ldots \ldots \ldots \ldots \ldots \ldots \ldots$

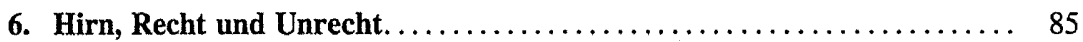

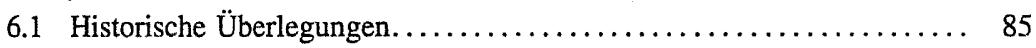

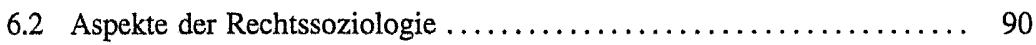

6.3 Erkenntnisse der Verhaltensforschung $\ldots \ldots \ldots \ldots \ldots \ldots \ldots \ldots \ldots . \ldots \ldots$

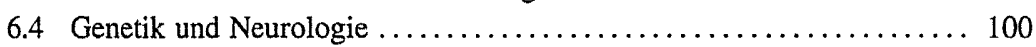

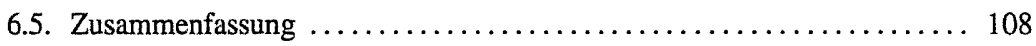

7. Exkurs: Neurologie und Strafrecht $\ldots \ldots \ldots \ldots \ldots \ldots \ldots \ldots \ldots \ldots \ldots \ldots$

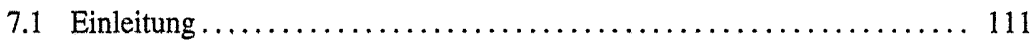

7.2 Strafrecht: Geschichte und Entwicklungen................. 113

7.3 Strafrecht: Massnahmen und Ziele...................... 118

7.4 Plädoyer für eine neue Strafrechtskonzeption $\ldots \ldots \ldots \ldots \ldots \ldots \ldots \ldots 121$

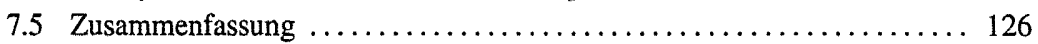

8. Neurologie und Menschenrechte......................... 129

8.1 Einführende Bemerkungen ............................ 129

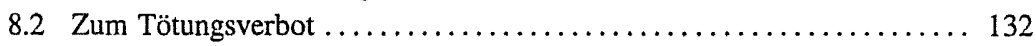

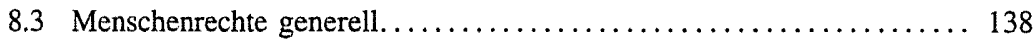

8.4 Folgerungen und Erkenntnisse. ........................ 144 
8.4.1 Propaganda versus Indifferenz.................... 144

8.4.2 Lernen und Emotionen ....................... 147

8.4 .3 Lernen durch Training.......................... 151

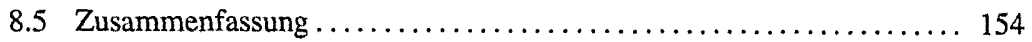

9. Menschenrechte und Rechtsstrukturen .................... 157

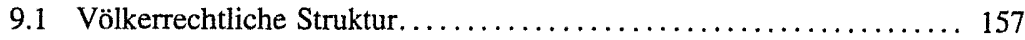

9.2 Nationalstaatliche Rechtsstruktur...................... 159

9.2.1 Legislative .............................. 159

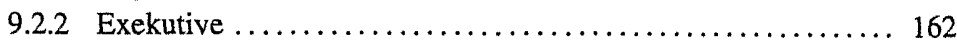

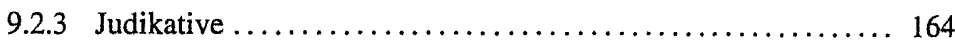

9.3. Die Transformation der Menschenrechte.................. 166

9.3.1 Eine neue Struktur der Menschenrechte ............... 166

9.3.2 Die individuellen Folgerungen $\ldots \ldots \ldots \ldots \ldots \ldots \ldots \ldots \ldots . \ldots \ldots$

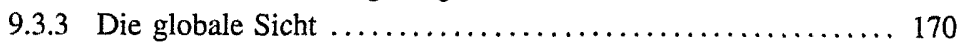

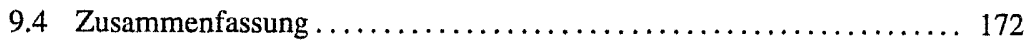

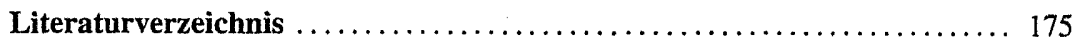

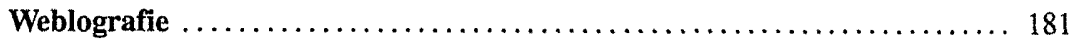




\section{Abbildungen und ihre Quellen}

Abbildung 2.1: Eleanor Roosevelt mit einem Poster der Menschenrechtserklärung, New York 1949

(C) F.D. Roosevelt Library..................... 25

Abbildung 3.1: Weltkarte zum Abstimmungsergebnis der Menschenrechtserklärung vom 10. Dezember $1948 \ldots \ldots \ldots \ldots \ldots \ldots \ldots \ldots, 41$

Abbildung 3.2: Weltkarte zum Pakt I beim Inkrafttreten am 3. Januar 1976. . 42

Abbildung 3.3: Weltkarte Pakt I am 1. Januar $1980 \ldots \ldots \ldots \ldots \ldots \ldots \ldots . \ldots 3$

Abbildung 3.4: Weltkarte Pakt I am 1. Januar $1990 \ldots \ldots \ldots \ldots \ldots \ldots \ldots . \ldots 4$

Abbildung 3.5: Weltkarte Pakt I am 1. Januar $2000 \ldots \ldots \ldots \ldots \ldots \ldots \ldots 44$

Abbildung 3.6: Weltkarte Pakt I am 1. Januar $2008 \ldots \ldots \ldots \ldots \ldots \ldots \ldots . \ldots 4$

Abbildung 3.7: Weltkarte zum Pakt II beim Inkrafttreten am 23. März 1976.. 46

Abbildung 3.8: Weltkarte Pakt II am 1. Januar 1980............... 46

Abbildung 3.9: Weltkarte Pakt II am 1. Januar 1990................ 47

Abbildung 3.10: Weltkarte Pakt II am 1. Januar 2000.............. 47

Abbildung 3.11: Weltkarte Pakt II am 1. Januar 2008............... 48

3.1-3.11 Quelle: http://www2.ohchr.org/english/bodies/ratification/3.htm\#N16 (5. März 2008) / ㄷ Grafik Halo

Abbildung 4.1: Anzahl der Publikationen in Schweizer Bibliotheken zum Stichwort Menschenrechte nach Erscheinungsjahren....... 59

Abbildung 4.2: Anzahl der Publikationen in der Deutschen Nationalbibliothek zum Stichwort Menschenrechte nach Erscheinungsjahren.

Abbildung 4.3: Anzahl der Publikationen beim Onlinebuchhändler Amazon zum Stichwort Menschenrechte nach Erscheinungsjahren

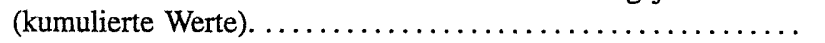

Abbildung 4.4: Anzahl der Beiträge in der New York Times mit dem Stichwort Menschenrechte im Titelbereich nach Jahren.

Abbildung 4.5: Anzahl der Beiträge in der Neuen Zürcher Zeitung mit dem Stichwort Menschenrechte im Titelbereich nach Jahren.......

Abbildung 4.6: Anzahl deutschsprachige Dokumente der Suchmaschine Google mit dem Stichwort Menschenrechte am 17. Juli 2008,

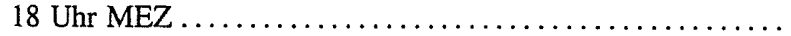

4.1-4.6 Quellen: im Text vermerkt / ㅇ Grafik Halo 
Abbildung 5.1: Urteile mit verminderter oder gar keiner Zurechnungsfähig-

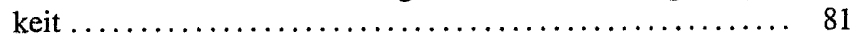

Abbildung 5.2: Urteile pro Jahr nach StGB in absoluten Zahlen........ 82

Abbildung 5.3: Strafurteile pro Jahr aufgrund sämtlicher Strafbestimmungen 83 5.1-5.3 Quelle: BfS/C Grafik Halo

Abbildung 6.1: Nationalsozialismus in Deutschland als Beispiel

(C) Corbis/Specter

Abbildung 6.2: Adolf Eichmann am Schreibtisch in seiner Zelle im RamleGefängnis in Tel Aviv 1961................. 89

(C) Keystone

Abbildung 6.3: Prägt das Recht den Menschen oder der Mensch das Recht? (C) Peter Rutkowski/Frankfurter Rundschau

Abbildung 6.4: Entwicklung des menschlichen Hirns

Quelle/C: http://www.joerg-peter-ewert.de

Abbildung 6.5: Das Erbrecht unter Verwandten.

Quelle/C: http://ruessmann.jura.uni-sb.de/rw20/wiwieinf/ wiwi5.gif

Abbildung 6.6: Das Experiment von Stanley Milgram.................

Quelle: Aus dem Film „Obedience“ (C) by Stanley Milgram; copyright renewed 1993 by Alexandra Milgram

Abbildung 6.7: Bild einer Nervenzelle im Hirn

(C) Eric Kandel, Columbia University, New York

Abbildung 6.8: Ein- und Ausschaltung von Genen in Hirnzellen

Quelle/C: http://www.joerg-peter-ewert.de

Abbildung 6.9: Der präfrontale Kortex.

(C) NEUROtiker, Creative Commons CC-BY-SA

Abbildung 7.1: Phrenologie

Quelle: Bilz, Friedrich Eduard (1842-1922): Das neue Naturheilverfahren (75. Jubiläumsausgabe), 1894

Abbildung 7.2: Mose zerschmettert die Gesetzestafeln ...

(C) Gustave Doré/Globalgallery.com

Abbildung 7.3: Folterszene auf einem anonymen Flugblatt aus dem 16. Jahrhundert, Quelle: Staschen, Heidi; Hauschild, Thomas: Hexen,

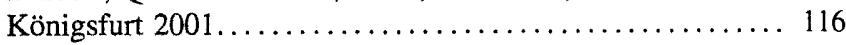

Abbildung 7.4: Formen strafrechtlicher Sanktionen $\ldots \ldots \ldots \ldots \ldots \ldots \ldots .120$ (C) Foto Halo

Abbildung 7.5: Menschliches Hirn

(C) Dieter Schütz (pixelio.de) 
Abbildung 8.1: Justizia und Neurologie

(C) Ole Vanhoefer

Abbildung 8.2: Massenhinrichtung in einem iranischen Gefängnis

(C) Keystone

Abbildung 8.3: Verhältnis der Todesopfer von Strassenverkehr und Gewaltverbrechen

Quelle: BfS/@ Grafik Halo

Abbildung 8.4: Waage als Symbol

(C) Grafik Halo

Abbildung 8.5: Der Fall Phineas Gage

Quelle: http://joeltalks.com/web_images/phineas_gage_ mcmillan.jpg itemId $=10408 \& \mathrm{~g} 2 \_$serialNumber $=1$

Abbildung 8.6: Stalin als Kapitän. 146

Quelle: http://www.hausblog14.ch/wp-content/uploads/ propaganda.jpg

Abbildung 8.7: Eric Kandel, Szenenfoto aus dem Film „Der Hirnforscher Eric Kandel“", Köln 2008, (c) FilmForm Köln GmbH........ 148 